\title{
Les chargés de communication dans les caisses d'allocations familiales. une professionnalité en émergence
}

Rosette Bonnet et Jacques Bonnet

\section{OpenEdition}

\section{Journals}

Édition électronique

URL : http://journals.openedition.org/communicationorganisation/2509

DOI : 10.4000/communicationorganisation.2509

ISSN : 1775-3546

Éditeur

Presses universitaires de Bordeaux

Édition imprimée

Date de publication : 1 mai 2001

ISSN : 1168-5549

Référence électronique

Rosette Bonnet et Jacques Bonnet, «Les chargés de communication dans les caisses d'allocations familiales. une professionnalité en émergence », Communication et organisation [En ligne], 19 | 2001, mis en ligne le 27 mars 2012, consulté le 19 avril 2019. URL : http://journals.openedition.org/ communicationorganisation/2509; DOI : 10.4000/communicationorganisation.2509

Ce document a été généré automatiquement le 19 avril 2019

(c) Presses universitaires de Bordeaux 


\title{
Les chargés de communication dans les caisses d'allocations familiales. une professionnalité en émergence
}

\author{
Rosette Bonnet et Jacques Bonnet
}

1 Depuis 1991, la Caisse nationale des allocations familiales (CNAF) a engagé la décentralisation de la fonction communication de sa branche Famille au niveau de ses organismes locaux: les Caisses d'allocations familiales (Caf). Dans ce contexte, la désignation de responsables de la communication a été réalisée dans la plupart de ces dernières. Ce sont aujourd'hui quelque 90 Chargé(e)s de communication (CC) qui sont en place dans les différents organismes locaux.

2 La recherche réalisée a pour objectif de caractériser les activités des Chargés de communication en CAF et de mettre en évidence le processus d'émergence de cette nouvelle professionnalité (Dubar. 1991), au sens d'une combinaison entre des aspects symboliques, notamment identitaires, et des aspects fonctionnels liés à l'exercice de la fonction communication. Cette professionnalité en devenir s'inscrit dans le contexte général de l'ouverture des administrations à des problématiques de communication, face à une demande sociale marquée par l'expression plus directe et plus intense des attentes des usagers des services publics en termes de disponibilité et de qualité. C'est l'illustration de cette émergence que nous proposons ici en cherchant également à produire des connaissances sur les savoirs professionnels requis des CC face aux exigences des situations auxquelles ils sont confrontés. Enfin, à partir de cette monographie, il s'agit d'engager une réflexion et une problématisation relatives aux modalités et au sens du changement organisationnel qui entoure l'apparition de la fonction communication dans les CAF et, plus globalement, d'esquisser d'autres pistes de recherche sur son développement dans les administrations françaises. 


\section{Méthodologie}

3 Nous avons fait le choix de préciser, au fil de l'exposé des résultats, la majeure partie des éléments qui ont servi de référents conceptuels à cette recherche.

4 Cependant, il convient de noter d'emblée que la collecte des données a été centrée, à travers l'appellation générique de Chargé(e) de communication, sur la problématique de «métier de communication en $\mathrm{CAF}$ » et donc-principalement sur les tâches et activités correspondantes. On entendra par métier un - ou des - ensemble(s) homogène(s) de savoirs professionnels (Dubar, 1998). L'agrégation de ces ensembles spécifie l'identité et la technicité des activités de communication dans les CAF. Du point de vue de l'analyse du travail (Zarifian. 1996), considérée ici en tant qu'appui méthodologique à cette recherche, la notion de tâche renvoie d'une part, à la prescription, c'est-à-dire aux attentes institutionnelles vis-à-vis de la fonction étudiée et, d'autre part, aux exigences de prise en charge, de réactivité et de traitement qu'impliquent les situations professionnelles rencontrées par les CC. Quant à lui, le concept d'activité concerne les opérations de travail effectivement réalisées par les personnels interrogés au cours des investigations.

5 Comme on le verra également dans l'analyse des résultats, ce métier pouvant revêtir des aspects statutaires et fonctionnels divers selon les organismes d'exercice, l'hypothèse de la forte hétérogénéité relative à la place, au rôle et à l'impact de la communication dans les $\mathrm{Caf}$ nous a conduits à considérer que si cette fonction existe bien en tant que telle dans ces organismes, elle fait l'objet de représentations (Jodelet, 1989) très variables et néanmoins influentes, quant à son positionnement institutionnel et à son image interne et externe. En ce sens, ce sont des logiques ou profils de métier que cette recherche vise à identifier et à caractériser en termes de convergences de représentations, de valeurs et d'attributions des acteurs, au regard de leur positionnement, de leurs tâches et de leurs activités (Doise, 1993 ; Dubar. 1998).

6 La collecte des informations a été effectuée à l'aide d'un questionnaire. Celui-ci a principalement permis une investigation au plan de la nature des activités et de l'identification des commanditaires des différentes productions, des difficultés et des contraintes qu'ils rencontrent, des compétences dont ils doivent, selon eux, faire preuve, ainsi que des habiletés qu'il leur paraît nécessaire de développer personnellement afin d'accroître leur efficience. En outre, il leur a été demandé de qualifier les activités de communication en CAF.

7 Soixante-cinq questionnaires ont été retournés et exploités. Le taux de questionnaires exploitables est particulièrement satisfaisant. Par ailleurs, les tests effectués montrent le très bon degré de significativité des réponses. Les données fournies par les réponses aux questionnaires ont également fait l'objet d'un approfondissement qualitatif fondé sur l'exploitation de dix entretiens semi-directifs. Les informations obtenues sur ce plan ont été intégrées dans les résultats ci-après. La validation de ces derniers a été effectuée à l'aide d'une nouvelle batterie de dix entretiens semi-directifs ainsi que d'une présentation des résultats devant le comité de pilotage de la recherche.

8 L'ensemble des informations recueillies a fait l'objet d'une analyse lexicale puis a été saisi, traité et exploité à l'aide du logiciel Sphinx 2000. Des documents de travail fournis par les CC ont permis une analyse complémentaire. Des expressions relevées au cours des entretiens et dans les questionnaires sont présentées entre guillemets. 


\section{Résultats}

\section{Caractérisation des activités et des productions des Chargés de communication}

Une première remarque s'impose : les activités de CC en Caf sont nombreuses, diverses et étendues. Trente-cinq modalités représentant les diverses activités et productions, ont fait l'objet de citations spontanées, ce qui est quantitativement important. Le registre lexical est riche puisque 454 citations ont été relevées, ce qui souligne encore le fait que chaque personne interrogée ait généralement mentionné sept à huit modalités.

Les activités de conception, de rédaction et de suivi de fabrication de documents et supports paraissent occuper une part importante des activités des CC. En particulier, ces derniers évoquent fréquemment le temps consacré à l'écriture en termes de fond et de forme. La dimension rédactionnelle et textuelle est donc prépondérante dans leur travail. $\mathrm{Au}$ delà des habituels "marronniers $~^{1}$, l'écriture est omniprésente dans les activités y compris lorsqu'elle se double de tâches de mise en page, de choix infographiques, d'adaptation à des supports variés, etc.

Tableau 1 - Activités et productions

\begin{tabular}{|c|c|c|}
\hline Activités et productions & Nb. cit. & Fréq. \\
\hline relation presse & 45 & $9.9 \%$ \\
\hline plans de communication & 43 & $9.5 \%$ \\
\hline rédactionnel & 34 & $7.5 \%$ \\
\hline collaboration parutions nationales & 33 & $7.3 \%$ \\
\hline plaquettes & 30 & $6.6 \%$ \\
\hline communication événementielle & 27 & $5.9 \%$ \\
\hline journal interne & 26 & $5.7 \%$ \\
\hline guides allocataires & 24 & $5.3 \%$ \\
\hline dossiers partenaires & 17 & $3.7 \%$ \\
\hline animation groupes/projets & 16 & $3.5 \%$ \\
\hline médias électroniques & 16 & $3.5 \%$ \\
\hline conception messages, maquettes & 15 & $3,3 \%$ \\
\hline Etudes, statistiques & 10 & $2.2 \%$ \\
\hline rapports activités & 10 & $2.2 \%$ \\
\hline management équipe communication & 9 & $2.0 \%$ \\
\hline formation. conseil & 8 & $1.8 \%$ \\
\hline réflexion stratégique & 8 & $1.8 \%$ \\
\hline multimédia films cd rom & 8 & $1.8 \%$ \\
\hline animation de réunions & 8 & $1.8 \%$ \\
\hline suivi fabrication supports & 7 & $1.5 \%$ \\
\hline choix prestataires & 7 & $1.5 \%$ \\
\hline chroniques radios locales & 7 & $1.5 \%$ \\
\hline documentation & 6 & $1,3 \%$ \\
\hline relationnel externe. & 6 & $1.3 \%$ \\
\hline revue de presse interne & 5 & $1.1 \%$ \\
\hline présence salons & 5 & $1.1 \%$ \\
\hline
\end{tabular}




\begin{tabular}{|l|c|c|}
\hline médiation interservices CAF & 5 & $1.1 \%$ \\
\hline projets interCAF & 4 & $0.9 \%$ \\
\hline rédaction discours direction & 3 & $0.7 \%$ \\
\hline participation Comdir & 3 & $0.7 \%$ \\
\hline mailing allocataires & 3 & $0.7 \%$ \\
\hline gérer crise & 2 & $0.4 \%$ \\
\hline cahiers charges. appels d'offres & 2 & $0.4 \%$ \\
\hline panneaux & 1 & $0.2 \%$ \\
\hline intervention écoles & 1 & $0.2 \%$ \\
\hline TOTAL CIT. & 4.54 & $100 \%$ \\
\hline
\end{tabular}

11 Le nombre de citations est supérieur au nombre d'observations du fait de réponses multiples (10 au maximum).

12 La dépendance est très significative. $\operatorname{chi} 2=387, \mathrm{ddl}=35, \mathrm{I}-\mathrm{p}=>99 \%$, Le chi2 est calculé avec des effectifs théoriques égaux pour chaque modalité. Les pourcentages sont calculés par rapport au nombre de citations.

13 En ce sens, les CC insistent sur la permanence du rapport à l'écrit dans leur métier, que ce soit à l'interne de l'organisme ou à l'externe. Les outils de bureautique tiennent donc une place importante dans la préparation, la gestion et le suivi des activités du CC, de même que les outils de communication électronique dont l'utilisation connaît une très forte croissance même si celle-ci apparaît inégale en termes d'impact et de satisfaction selon les lieux et les acteurs. L'articulation entre d'une part l'écriture et, d'autre part, la réflexion et la conception de messages, s'opère notamment sur des objets comme la collaboration aux parutions nationales CNAF, la réalisation de plaquettes et de guides destinés aux allocataires, la rédaction de comptes rendus, de rapports d'activités, la préparation de mailing, etc. Elle se traduit aussi dans la préparation et la gestion de messages destinés à l'ensemble des moyens télématiques et des médias électroniques que les CAF développent actuellement.

14 Les relations avec les médias, et notamment avec le média Presse, constituent une autre dominante des activités des CC. La modalité «relations presse » obtient le plus grand nombre de citations (45), traduisant ainsi également la place, voire parfois les interrogations, que représente cette activité chez les CC. La préparation de dossiers, de communiqués, les interventions face à des journalistes, sont, comme les entretiens semidirectifs ont permis de le préciser, des domaines d'activités qui constituent très nettement, aux yeux des $\mathrm{CC}$, des enjeux forts liés à leur fonction, notamment en termes de représentation de leur organisme d'appartenance et de porteurs de messages vers l'ensemble des usagers et partenaires. En ce qui concerne ces derniers, il s'agit essentiellement, au plan interne, de la direction, des administrateurs, des cadres et autres personnels des Caf. Au plan externe, les interventions des CC se font en relation avec des organismes sociaux, des collectivités territoriales et des associations.

15 Les activités mentionnées sous la modalité "plan de communication » font également l'objet 43 citations. Elles renvoient à une représentation plus globale, plus systémique davantage structurée en étapes et en procédures de réflexion, de conception, de mise en 
œuvre et d'évaluation de messages. Sont mentionnés dans le cadre de cette modalité tant des plans à vocation d'orientation globale et/ou stratégique au niveau local, que des actions ponctuelles, par exemple en direction des allocataires. Il s'agit alors de dossiers dont l'importance nécessite une organisation, une réflexion et une logistique spécifiques. À titre d'exemple et sans généralisation trop hâtive, il peut s'agir d'une campagne de sensibilisation des étudiants en ce qui concerne leurs droits vis-à-vis de certaines prestations. Notons que la fonction d'évaluation et de prospective ou différée des actions de communication engagées est relativement peu présente dans les activités décrites par les CC. Elle est cependant mentionnée à 10 reprises sous le vocable d'études ou de statistiques. Il est encore possible de relever que, si les CC évoquent bien les deux grands registres d'activités des $\mathrm{CAF}$, c'est-à-dire en les résumant rapidement les prestations et l'action sociales, ils envisagent généralement leurs activités de communication d'un point de vue global en tant que métier spécifique devant être capable de s'emparer des multiples objets liés à la communication et relevant de ces deux branches. Certains ont cependant mentionné le fait qu'ils étaient davantage missionnés sur l'une d'elles.

La communication événementielle apparaît également comme un bloc d'activités important pour les $\mathrm{CC}$ car répondant tant à des besoins d'identité, de mise en synergie, de partage d'informations et de convivialité au plan interne, qu'à la nécessité de construire et de développer une image externe favorable et porteuse d'intérêt auprès des différentes catégories de cibles. La prise en charge totale ou partielle de journaux internes est à associer avec les résultats obtenus pour la modalité «revue de presse interne" l'ensemble de ces données marquant le rôle du CC en matière d'information interne à l'organisme.

La modalité «dossiers partenaires» doit être combinée avec la modalité «relationnel externe ». Toutes deux expriment des activités de contacts pérennisés, de lobbying. de concertation et de coopération professionnelle, de prise d'avis et de conseils, d'apports d'informations ayant également parfois valeur de diplomatie et d'attention portée à ces partenaires individuels ou collectifs. Ces relations externes ou relations publiques sont encore complétées parfois par la présence du CC sur certains salons ainsi que lors d'interventions dans des écoles à vocation de professionnalisation, par exemple auprès de futurs Travailleurs sociaux ou Cadres de santé.

Les activités managériales s'appliquent à la participation et à l'impulsion, au pilotage et à l'évaluation de projets et de plans de communication sous la conduite, ici aussi exhaustive ou partielle, du CC. Elles concernent encore le management de l'équipe ou du service communication lorsque ces derniers existent dans l'organisme en tant qu'entité propre. Cela indique également une diversité assez importante, selon les CAF, en ce qui concerne le statut et les responsabilités attribuées, le positionnement dans l'organigramme, les missions et les résultats attendus, les ressources humaines et matérielles affectées, etc.,

19 L'animation d'équipes et de réunions est pointée comme un autre champ d'activités plaçant le CC au cœur d'une fonction de médiation, d'intervention et de conseil interne, de facilitation de l'expression et de la production de groupes travaillant à des objectifs et à des tâches qui ne sont pas nécessairement liés à des problématiques de communication.

20 Sur la base de recherche de corrélations entre les activités et les difficultés rencontrées, on remarquera, comme les entretiens semi-directifs l'ont également confirmé, que lorsque les personnes interrogées déclarent effectuer des activités de formation, de conseil interne ou encore de gestion des ressources humaines, elles se trouvent 
fréquemment affectées à temps partiel, c'est-à-dire à un tiers, voire un quart-temps et même parfois moins, à la fonction communication au sein de leur organisme.

Quelques citations évoquent une part stratégique dans les activités réalisées. Lors des entretiens, a été mentionné à plusieurs reprises un écart entre des lettres de missions qui, lorsqu'elles existent, prescrivent au CC une intervention et des préconisations de niveau stratégique, alors que la réalité de leurs activités semble généralement laisser moins de place à cette dimension. Le CC est également un homme ou une femme susceptible d'assurer une fonction de constitution et d'animation de réseaux formels ou informels entre différentes $\mathrm{Caf}$, par exemple dans le cadre de projets dépassant l'intervention locale d'un organisme particulier. La gestion de situations de crise, notamment au plan externe en ce qui concerne des dysfonctionnements informationnels ou bien au plan interne à propos de difficultés interpersonnelles et de groupes, a été assez fréquemment évoquée lors des entretiens semi-directifs alors qu'elle ne fait l'objet que de deux citations dans les réponses issues du questionnaire.

\section{Un métier, des logiques}

L'ensemble de ces résultats laisse apparaître différentes logiques quant à l'exercice des activités liées à la communication au sein des Caf, tout comme il révèle deux axes de professionnalité.

- La première logique est aussi la plus importante et la plus fréquente. Elle s'appuie sur un rapport à la fois généraliste et direct à l'action de production, notamment en termes de conception et de fabrication de messages. Elle prend fortement appui sur les évolutions technologiques concernant la création et la transmission des informations. Elle se manifeste également au plan d'une ingénierie et d'une gestion des ressources et des moyens de communication destinés à diverses manifestations de type événementiel. Davantage positionnée comme une fonction technique ayant un rapport modéré à la conception et à l'impulsion de stratégies de communication, elle apparaît largement majoritaire en termes de profil de métier au sein des Caf. Dans ce contexte d'évolutions technologiques, on observe, à travers les discours et les écrits de quelques CC, la place et les rôles parfois quasi exclusifs qu'ils attribuent à la bureautique et à la communication électronique en tant que caractéristiques de leur métier et de leur efficience. Ce constat interroge, en termes de grandes orientations et de missions, la professionnalité liée aux activités de communication en Caf.

- Une seconde logique représente une approche davantage Managériale de la communication. Elle est souvent liée à une position d'encadrement que celle-ci soit validée ou non au plan statutaire. On peut également rencontrer cette logique dans le cas où la fonction de communication est assurée par une seule personne. Dans ce contexte, les activités consistent surtout en une mise en synergie des compétences et des ressources autour de projets et de plans ponctuels ou plus globaux et plus fondamentaux du type "projet d'établissement ou projet de service». Dans les Caf, des distinctions semblent opérées avec plus ou moins de formalisme et de conviction, soit entre la communication interne et la communication externe, soit entre les deux modes de communication propres aux grandes branches traditionnelles (famille, maladie), soit, parfois, simultanément sur ces deux plans. On peut faire ici l'hypothèse que lorsque la première de ces disjonctions se trouve avérée, elle n'est pas sans effets au plan identitaire interne ainsi qu'au plan de l'image portée à l'externe par l'organisme concerné. 

écrites et les propos des personnes interrogées. Elle concerne une posture d'analyste, d'observateur qui confine à la fonction de chargé d'études ou de mission faisant l'objet d'un mandat plus ou moins explicite en la matière. D'un point de vue général, les missions, objectifs et résultats assignés à la communication demeurent relativement implicites dans le cadre de cette fonction de " préparateur ».

26 - Certains CC décrivent encore leurs activités sous un angle davantage expérimental, se référant ainsi à une représentation d'interpellation, voire de "poil à gratter » en ce qui concerne la fonction communication au sein de l'organisme.

27 - À travers certaines citations, c'est, enfin, davantage une fonction d'information, plus que de communication, qui est évoquée, par exemple par des activités de gestion de fond documentaire, de revues de presse, d'abonnements. Dans quelques cas, cette logique est apparue prépondérante au sein de l'organisme.

\section{Deux axes de professionnalité}

28 En synthèse de ces différentes logiques de métiers, deux conceptions de la professionnalité de $\mathrm{CC}$ peuvent finalement être distinguées à propos des représentations portées sur leurs missions et sur leurs activités.

29 L'une aborde le métier de CC prioritairement à l'aune du service rendu aux allocataires et aux personnels, ce qui induit la primauté d'une proximité sociale et technique à l'égard de ces derniers. Cette axiologie semble se référer à une culture du travail social, activité que de nombreux CC ont traversée. La seconde considère la fonction de CC comme relevant essentiellement d'un rapport de proximité avec les technologies d'information et de communication, les médias et les relations externes.

30 Enfin, les questionnaires et les entretiens semi-directifs révèlent que si une part non négligeable des CC indiquent qu'ils ont reçu une formation en communication plus ou moins étendue et adaptée, nombreux sont ceux qui déclarent avoir intégré ces activités « par la bande » ou au hasard de leur parcours professionnel, ce qui les conduit parfois à éprouver le sentiment «d'être novices » vis-à-vis de leur métier, y compris après plusieurs années d'exercice. De ce point de vue, nous retiendrons le besoin de référents institutionnels, professionnels et symboliques qui caractérise une professionnalité encore mal définie et donc peu assurée tant vis-à-vis d'elle-même que de l'institution où elle s'exerce.

\section{Difficultés et contraintes évoquées par les Chargés de communication}

31 Les difficultés et contraintes liées aux activités de communication font l'objet de 239 citations (cf. tableau 2). Les évocations sont assez ciblées et précises. Elles manifestent un fort niveau de cohérence avec les activités déjà décrites et les champs de compétences qui seront analysés ultérieurement.

Communication et organisation, 19 | 2001 


\begin{tabular}{|l|r|r|}
\hline Difficultés et contraintes & Nb. cit. & Fréq. \\
\hline travail dans urgence & 40 & $16.7 \%$ \\
\hline Chargé de com peu associé à réflexion. stratégic & 2.3 & $9.6 \%$ \\
\hline positionnement Communication/CAF & 19 & $7.9 \%$ \\
\hline fonction communication : I personne/temps particl & 18 & $7.5 \%$ \\
\hline manque intérêt autres services/cadres & 14 & $5.9 \%$ \\
\hline évaluer impact actions & 12 & $5.0 \%$ \\
\hline manque moyens, matériel & 11 & $4.6 \%$ \\
\hline nombreuses actions parallèles & 10 & $4.2 \%$ \\
\hline besoin formation permanente & 9 & $3.8 \%$ \\
\hline méconnaissance interne impact Communication & 8 & $3.3 \%$ \\
\hline dépendance outils informatique & 7 & $2.9 \%$ \\
\hline quête permanente d'informations & 7 & $2.9 \%$ \\
\hline gérer diversité intervenants/supports & 6 & $2.5 \%$ \\
\hline exigence croissante qualité. professionnalisme & 6 & $2.5 \%$ \\
\hline segmentation Communication selon allocataires & 6 & $2.5 \%$ \\
\hline relations presse & 6 & $2.5 \%$ \\
\hline fonction mal définie & 5 & $2.1 \%$ \\
\hline insuffisance liens management/Com & 5 & $2.1 \%$ \\
\hline mauvaise communication interne CAF & 5 & $2.1 \%$ \\
\hline rétention d'informations & 4 & $1.7 \%$ \\
\hline frilosité acteurs changement & 4 & $1.7 \%$ \\
\hline commandes peu claires & 4 & $1.7 \%$ \\
\hline budgets imprécis & 3 & $1.3 \%$ \\
\hline coordination service informatique & 3 & $1.3 \%$ \\
\hline communication perçue fourre-tout & 2 & $0.8 \%$ \\
\hline nécessité se légitimer en permanence & 1 & $0.4 \%$ \\
\hline réunir les acteurs & $0.4 \%$ \\
\hline TOTAL ClT. & & $100 \%$ \\
\hline
\end{tabular}

Le rapport au temps dans le travail constitue, selon les CC, une source importante de difficultés et de contraintes dans leurs activités. Sur ce plan, la notion d'urgence est omniprésente. Répondre, se mobiliser, réagir dans des délais très courts à des exigences fortes, semble être l'apanage de la fonction. La nécessité d'articuler de nombreuses actions et interventions simultanées ou, tout au moins, sur des pas de temps limités et proches, conforte ce sentiment permanent d'urgence, voire de retard qui engendre, selon les interviewés, des effets de culpabilisation et de stress. Cette difficulté identitaire est également souvent liée au fait que, au sein des Caf, la fonction de communication serait, aux yeux des CC, peu associée et intégrée au montage des dossiers, plans et projets et, plus globalement, à la réflexion préalable en amont de son intervention. Les expressions de "dépannage, cerise sur le gâteau, pompier de services, enjoliveur, fourre-tout, dernière carte en cas de pépin ou de crise » ont été fréquemment prononcées même si elles se trouvent parfois tempérées par les interviewés qui relatent également des situations de réussite.

Ici émerge donc une problématique clé de la fonction de communication en Caf. Elle concerne son positionnement, lequel fait l'objet de nombreuses citations en termes de manque d'intérêt, notamment de la part de l'encadrement et des personnels des autres services, de méconnaissance de son potentiel et de son impact, d'absence de liens avec le management interne, de rétention d'informations, d'image floue et ludique, etc. Ce sont autant de handicaps qui nécessitent, selon les CC, une quête permanente d'informations et de données valides et actualisées ainsi qu'une démarche de légitimation de tous les instants. Il est clair que, selon qu'elle se trouve rattachée à la Direction générale, organisée en "pool » interservices, constituée en service indépendant, identifiée, dotée ou non de ressources humaines et de moyens matériels, la fonction de communication n'est pas régie et fondée sur les mêmes principes et les mêmes objectifs de résultats. 

prenant en compte le fait que les $\mathrm{CC}$ expriment parfois un message en direction de leur institution à travers leurs dires, elle ne peut par trop ignorer la fréquence et la force de ces remarques, notamment au plan de leur argumentation. Ainsi, la fonction de communication en Caf semble souffrir d'une forte difficulté identitaire qui se conjugue avec des difficultés fonctionnelles bien que, paradoxalement, l'on fasse de plus en plus appel à elle... surtout dans l'urgence.

Notons encore en liaison avec les constats énoncés ci-dessus que les $\mathrm{CC}$ déclarent recevoir et vivre des exigences de plus en plus fortes, à l'interne comme à l'externe, au plan du professionnalisme et de la qualité des services et des productions qu'ils ont à rendre. Cela semble représenter une manière implicite de souligner l'importance de la fonction communication, tout au moins dans sa capacité à répondre rapidement à des besoins de production ou de remédiation, mais moins en termes d'intégration à la dimension stratégique de l'organisme.

citations évoquent encore une relative frilosité des acteurs, et notamment de l'encadrement, face au changement de perception proposé par la communication à propos de l'organisation du travail, du management des plans et projets, des modalités de contact avec les publics et les partenaires, etc.. La communication, champ scientifique et technique interdisciplinaire (Wolton. 1997), semble se heurter à des conceptions et des territoires idéels qui constituent autant de logiques disjonctives au sein de l'organisation étudiée. Les problèmes d'identité et de légitimité semblent entretenus par le fait que la fonction communication éprouve des difficultés à s'appuyer sur l'évaluation comme mode de pilotage et de validation de ses résultats. qu'avec l'ensemble des intervenants et prestataires, le fait d'être confrontés avec des demandes et des commandes parfois peu explicites à l'interne comme à l'externe, l'imprécision des enveloppes budgétaires affectées aux différents projets et actions de communication, la quasi impossibilité de réunir les personnels et les partenaires concernés par une même action, constituent d'autres questions vis-à-vis desquelles les personnes interrogées déclarent éprouver des difficultés. Il en est de même en ce qui concerne l'objectif de segmentation et donc d'adaptation des messages et des supports selon les allocataires et les publics. Sur ce dernier plan, c'est bien une difficulté de conception et d'intervention stratégique qui est pointée. infographie et de toutes formes de communication électronique, est reconnue par les CC comme indispensable à leur efficience professionnelle, certains d'entre eux éprouvent des difficultés pour dépasser, selon leurs propres termes, «le stade du bricolage » et du « système $\mathrm{D}$ » ou encore de la « débrouillardise » en la matière. Les efforts d'animation, de formation et d'information engagés sont soulignés par les interviewés mais ils notent dans le même temps les problèmes importants qu'ils rencontrent au quotidien. À titre d'exemple, la communication électronique est perçue comme très utile et pouvant faciliter le travail individuel et en réseau ainsi que comme facteur d'aide à la décision. Par contre, elle est, dans le même temps, décrite comme actuellement trop instrumenlalisée, lourde, particulièrement "chronophage " et, paradoxalement, comme une barrière de communication avec les collègues et les services internes en raison de l'effort de sélection d'information qu'elle exigerait. Le temps consacré, notamment le matin lors de la prise de poste, à distinguer les informations et dossiers urgents et sensibles de ceux moins 
pressants, à prioriser la note interne à valeur de consigne ou bien la commande de travaux par rapport à l'information générale leur paraît excessif. Ce temps de lecture et de traitement semble poser de réels problèmes, surtout lorsque les effectifs et les moyens de la fonction communication sont réduits. C'est donc là également un problème de diffusion, de segmentation et d'accès à des niveaux d'information différents qui est posé, ainsi que la nécessité de développer des habiletés de priorisation et d'organisation chez les destinataires qui soulignent cependant l'intérêt professionnel des différents moyens de communication électronique. La question de la technologie en tant qu'outil/support ou finalité ne parait pas encore pleinement clarifiée et la qualité technique d'un dispositif ne suffit sans doute pas pour surmonter, en tous les cas à court et moyen termes, des difficultés relatives à des aspects culturels, cognitifs et notamment procéduraux chez les utilisateurs. La problématique énoncée ici relève des modèles de changement dans la manière de travailler. Elle interroge également l'appropriation de nouveaux modes de diffusion et d'exploitation de l'information, fondés sur des habiletés accrues de discernement, de priorisation et d'adaptabilité des messages. Bien sûr, cette remarque ne concerne pas les seuls $\mathrm{CC}$, ni les seules $\mathrm{Caf}$, tant elle pointe les problèmes rencontrés du fait de l'exigence de réactivité immédiate qu'exige la communication électronique fondée sur le mythe de la transparence et de l'ubiquité (Wolton, 1997), lequel fait souvent fi des besoins des interlocuteurs en matière de distanciation réflexive et d'appropriation des messages émis...

39 Ainsi, chez certains $\mathrm{CC}$ et quelles que soient les bonnes volontés à son égard, la communication électronique paraît occuper des plages de temps démesurées au regard des autres activités à conduire, ce qui pose le problème de son intégration dans les activités. Pour d'autres, des freins, des inhibitions demeurent en termes de distance culturelle vis-à-vis des technologies d'information et de communication. Sans généralisation abusive, une pédagogie du changement du travail en communication paraît attendue afin d'éviter les dérives organisationnelles de la " Totale Technologie » ou à l'inverse du blocage culturel envers les TIC, aussi infructueux l'un que l'autre en termes d'identité et de professionnalisation. Les CC évoquent eux-mêmes ce besoin de formation et d'adaptation permanentes aux différentes facettes de-leur métier dont ils notent, par ailleurs, la " complexité croissante ». Enfin, les relations entre la fonction communication et les services informatiques des Caf paraissent parfois difficiles en raison de conceptions différentes de leurs interventions respectives et dénotent ainsi des chocs de cultures dans le travail (Camilleri. 1989). Selon les personnes interrogées, les services informatiques raisonnent "par le global, par la nature des équipements et des fonctionnalités », la communication, quant à elle, chercherait à s'exprimer davantage en termes de « messages, de services, de rapport aux hommes et au champ du social en général ».

\section{Les représentations de la compétence professionnelle chez les Chargés de communication}

40 Le tableau 3 montre, à travers le nombre de citations obtenues (301) l'intérêt des CC vis-àvis de la question des compétences requises pour l'exercice de ce métier. Précisons que nous entendons ici par compétence, la possibilité individuelle ou collective de construire, de mobiliser, de combiner et d'appliquer des savoirs en réponse à une situation professionnelle (Bonnet. 1998). Le premier champ de compétences mis en avant par les CC concerne des savoirs professionnels relatifs à des habiletés d'expression écrite et orale, 
lesquelles recueillent plus de $13 \%$ des citations, confirmant ainsi l'importance des activités de rédaction et d'intervention en public. De même se trouve pointée la capacité d'adaptation à différents interlocuteurs dans un cadre-individuel ou collectif. Celle-ci est encore soulignée par 18 citations concernant l'aptitude à découvrir et comprendre les attentes et besoins des publics et partenaires. Ce sont donc finalement 52 citations qui renvoient à des habiletés liées à l'interculturalité, au sens selon lequel la capacité à identifier les systèmes de représentations, de valeurs, d'attributions, chez autrui, constitue une facette importante de la compétence des CC. Celle-ci s'exprimerait donc en termes d'élaboration d'une communication interpersonnelle propice aux interactions et à la production d'une intelligibilité réciproque (Clanet, 1993) dans le travail interne et externe à l'institution.

Tableau 3 - Compétences nécessaires au Chargé(e) de communication

\begin{tabular}{|c|c|c|}
\hline Compétences & Nb. eit. & Fréq. \\
\hline expression écrite/orale & 41 & $13.6 \%$ \\
\hline adaptabilité & 34 & $11.3 \%$ \\
\hline connaissance activités/produits & 21 & $7.0 \%$ \\
\hline organiser & 21 & $7.0 \%$ \\
\hline découverte attentes/hesoins & 18 & $6.0 \%$ \\
\hline maîtrise bureautique PAO & 17 & $5.6 \%$ \\
\hline comprendre stratégie organisme & 15 & $5.0 \%$ \\
\hline esprit de synthèse & 1.5 & $5.0 \%$ \\
\hline consciller clairement & 12 & $4.0 \%$ \\
\hline créativité & 12 & $4.0 \%$ \\
\hline réactivité & 11 & $3.7 \%$ \\
\hline disponibilití/veille & 10 & $3.3 \%$ \\
\hline management projets & 9 & $3.0 \%$ \\
\hline animation équipes & 7 & $2.3 \%$ \\
\hline chaîne graphique & 7 & $2.3 \%$ \\
\hline adaptation supports/cibles & 6 & $2.0 \%$ \\
\hline utilisation médias & 6 & $2.0 \%$ \\
\hline évaluer résultats & 5 & $1.7 \%$ \\
\hline objectivité/diplomatie & 5 & $1.7 \%$ \\
\hline méthodologie d'enquêtes & 4 & $1.3 \%$ \\
\hline identifier orientations politiques & 3 & $1.0 \%$ \\
\hline intégrer contraintes/critiques & 3 & $1.0 \%$ \\
\hline négocier & 3 & $1.0 \%$ \\
\hline suivi actualité locale & 2 & $0.7 \%$ \\
\hline relation positive Direction & 2 & $0.7 \%$ \\
\hline rencontrer allocataires & 2 & $0.7 \%$ \\
\hline autonomie & 2 & $0.7 \%$ \\
\hline Iravail équipe/concertation & 2 & $0.7 \%$ \\
\hline aptitude au changement & 2 & $0.7 \%$ \\
\hline travail en réseaux & 1 & $0.3 \%$ \\
\hline marketing & 1 & $0.3 \%$ \\
\hline cahiers des charges & 1 & $0.3 \%$ \\
\hline statistiques & 1 & $0.3 \%$ \\
\hline TOTAL CIT. & 301 & $100 \%$ \\
\hline
\end{tabular}

41 D'autres compétences sont mentionnées. Il s'agit notamment de manifester un esprit de synthèse, d'être «capable de détecter et d'émettre l'essentiel ». à l'interne ou lors des relations avec les médias, ou encore d'effectuer des recommandations, des préconisations claires, structurées, argumentées et cohérentes en termes de faisabilité et d'opérationnalité concernant des actions ponctuelles ou des plans de communication. Cette capacité à "penser système et non saupoudrage" semble s'appliquer particulièrement aux compétences attribuées à l'animation et au pilotage de plans et de projets de communication.

42 Être capable de comprendre et de s'insérer dans la politique de son organisme d'appartenance, être capable de le représenter, autrement dit savoir identifier et intégrer dans l'action les orientations stratégiques d'une Caf, recueille 15 citations explicites. Si l'on croise ce type de réponse avec les résultats obtenus dans les autres questions et au cours des entretiens semi-directifs, on constate l'importance que les CC accordent à la 
dimension politique de leur métier, même s'ils éprouvent des difficultés à en formuler les contours précis et les effets. Ils perçoivent et comprennent le rôle de cette dimension à travers l'expérience issue de leur action quotidienne et la considèrent comme primordiale tout en émettant le souhait d'une part, d'y être davantage associés au plan global et, d'autre part, de disposer de bases conceptuelles et méthodologiques plus solides leur permettant de mieux répondre à cette nécessité lors de leurs diverses interventions. Une bonne connaissance de l'ensemble des métiers exercés en Caf, des activités et des services offerts par l'organisme constitue, comme les entretiens l'ont confirmé, un socle essentiel pour l'efficience professionnelle. Certains CC ont déclaré se sentir insuffisamment informés et préparés à ce sujet. Cette difficulté à disposer de temps et de moyens pour approfondir la compréhension des métiers et des productions est à relier avec les problèmes d'urgence de fabrication et de réactivité évoqués ci-dessus.

Les réponses mentionnent également l'importance des compétences en matière de bureautique, de PAO, d'utilisation de la communication électronique, mais de façon plus réservée que lorsqu'il s'agissait d'évoquer l'exercice quotidien des activités. Un certain déplacement s'opère donc ici vers l'image d'un professionnel maîtrisant prioritairement l'expression orale, la rédaction, la conduite de projets et d'équipes, la négociation et la médiation la réflexion stratégique, la relation aux médias, l'organisation des activités. Ce décalage est donc identifiable entre d'une part, le métier tel qu'il est décrit à travers des activités quotidiennes fortement liées à la conception et à la fabrication de messages, et, d'autre part, la représentation que projettent les CC en termes de compétences requises. Ainsi, c'est une professionnalité davantage liée à la communication sous ses formes interpersonnelles, institutionnelles et médiatiques qui est dépeinte comme caractéristique de leur fonction, au détriment, certes relatif, des activités de production de messages et supports et de traitement d'information pourtant prédominantes dans leur quotidien et davantage fondées sur l'usage des nouvelles technologies. Ici encore, deux logiques professionnelles émergent donc dans les réponses obtenues, l'une globalement liée à des fonctions de secrétariat et/ou de fabrication, l'autre plutôt relative à des activités de conception ainsi que de relations internes et externes. Cependant, dans le contexte du terrain et de l'action, ces deux logiques semblent parfois peu clarifiées.

Les entretiens confirment à nouveau ici la très grande disparité d'utilisation et de maîtrise des $\mathrm{CC}$ vis-à-vis des technologies d'information et de communication. L'écart relatif relevé ici entre une représentation idéalisée du métier et les activités auxquelles il donne effectivement lieu, interroge l'emploi de CC au plan de ses enjeux, de ses missions, de son positionnement et des savoirs professionnels nécessaires à son exercice, notamment en ce qui concerne le rapport et l'équilibre entre technique, technologie, intervention interne, représentation externe et stratégie de communication. Il est important d'ajouter que ce sont principalement des savoirs déclaratifs (connaissances) et des savoirs procéduraux (processus de raisonnement, d'action et de comportement) qui sont mis en avant par les CC en tant que compétences requises essentielles à l'exercice de leur métier. Ils voient dans ces deux catégories de savoirs les conditions de construction, de consolidation et de développement de leur professionnalisme ainsi que de l'identité du $\mathrm{CC}$ en Caf. Globalement, la recherche montre un déficit relatif au plan de ces savoirs professionnels complexes que sont les savoirs procéduraux en termes de capacités à diagnostiquer, problématiser, raisonner stratégie, évaluer... On retrouve ce besoin par exemple dans l'importance accordée à la capacité d'anticiper et d'évaluer des (et ses) 
résultats. Enfin, le $\mathrm{CC}$ doit encore, selon ses pairs, être réactif, disponible, en état de veille, en prise permanente avec l'actualité.

\section{Compétences que les Chargés de communication souhaitent et estiment nécessaire de développer}

Tableau 4 - Compétences ci développer

\begin{tabular}{|l|c|c|}
\hline Compétences à développer & Nh. cit. & Fréq. \\
\hline Communication électronique & 25 & $1.3 .0 \%$ \\
\hline PAO/infographie & 21 & $10.9 \%$ \\
\hline concevoir et évaluer plan de communication précis & 20 & $10.4 \%$ \\
\hline management équipes et projets & 18 & $9.3 \%$ \\
\hline élaboration stratégie Communication & 16 & $8.3 \%$ \\
\hline répondre aux médias, interviews & 15 & $7.8 \%$ \\
\hline argumentation/négociation/médiation & 11 & $5.7 \%$ \\
\hline expression en public & 11 & $5.7 \%$ \\
\hline gérer relations presse & 9 & $4.7 \%$ \\
\hline bureautique & 7 & $3.6 \%$ \\
\hline manager équipe Communication & 5 & $2.6 \%$ \\
\hline statistiques, enquête & 5 & $2.6 \%$ \\
\hline rédactionnel & 4 & $2.1 \%$ \\
\hline story-board, scénarios & 3 & $1.6 \%$ \\
\hline créativité & 3 & $1.6 \%$ \\
\hline prises de vues photo vidéo & 3 & $1.6 \%$ \\
\hline communication événementielle & 3 & $1.6 \%$ \\
\hline communication urgence et crise & 2 & $1.0 \%$ \\
\hline gérer adresses et références & 2 & $1.0 \%$ \\
\hline journal interne & 2 & $1.0 \%$ \\
\hline gestion du temps & 2 & $1.0 \%$ \\
\hline connaissance des prestations, des produits & 2 & $1.0 \%$ \\
\hline animer manifestations institutionnelles/débats & 2 & $1.0 \%$ \\
\hline réaliser films & 1 & $0.5 \%$ \\
\hline adapter intranet & 19.3 & $100 \%$ \\
\hline TOTAL CIT. & & \\
\hline & 3 & $2.5 \%$ \\
\hline & $2 \%$ & $2 \%$ \\
\hline
\end{tabular}

Le nombre de citations est d'un tiers moindre par rapport à la question précédente (193 contre 301). Pourtant, l'enseignement apporté ici est riche et nourrit la compréhension des paradoxes déjà évoqués à propos du positionnement et des savoirs professionnels attribués à la fonction de communication. Ainsi, les besoins de compétences solides au quotidien en matière de communication électronique, de PAO et. à un degré moindre, de bureautique, sont fortement cités. Ces réponses correspondent à «l'urgence technologique » ainsi qu'à «la production croissante de supports » et à la nécessité de «travailler en concertation et en réseaux internes et externes». Elles manifestent leur intérêt et, le plus souvent, leur adhésion envers les nouvelles technologies malgré certaines difficultés déjà évoquées au plan quotidien.

Une autre catégorie importante de besoins se manifeste. Elle concerne l'acquisition ou la consolidation d'une culture et de savoirs professionnels en communication permettant aux CC de mieux maitriser la conception, le pilotage et l'évaluation de plans et de projets au niveau opérationnel comme au niveau stratégique. Ce sont encore des compétences d'ordre systémique qui sont appelées ici, elles renvoient essentiellement à des savoirs de processus concernant l'analyse et le diagnostic, l'identification de problématiques dans les informations et les messages, la réflexion et la conception stratégique, le pilotage par l'évaluation, etc.. Savoir comment s'y prendre et comment faire pour partager une 
culture communicationnelle, pour manager une équipe, comprendre et/ou construire une orientation politique en Caf à l'aide de scénarios, la transférer, l'expliquer, la faire partager à l'interne et la traduire dans des messages et des supports adaptés aux publics et aux conditions partenariales constituent quelques représentations de la professionnalisation souhaitée par les CC.

Il apparaît que la notion de projet et de plan de communication ne renvoie pas chez les intéressés à des pratiques, à des processus et à des procédures suffisamment clairs, stabilisés et formalisés, ce qui les conduit parfois à se considérer «trop peu spécialistes, experts ou professionnels » face à cette tâche dont ils mentionnent par ailleurs l'importance.

\section{Représentations de la communication en CAF}

Plusieurs catégories de représentations apparaissent dans les résultats. La première (35 citations) concerne l'image de complexité attribuée aux activités de communication en Caf. Les personnes interviewées insistent notamment sur la multiplicité et la multiréférentialité de leurs travaux. Elles fournissent non pas une image de flou, de fourre-tout globalisant, mais plutôt la silhouette d'un métier en émergence et de pratiques professionnelles qui font appel à de multiples sources d'information et d'interlocuteurs. C'est donc une diversité de tâches qui est identifiée et qui semble mobiliser des habiletés en matière de « zapping informationnel » dans un contexte et des activités marqué par une polychronie sociale et cognitive (Camilleri \& Vinsonneau. 19\%).

Tableau 5 - Qualificatifs caractérisant la communication en CAF. selon les CC

\begin{tabular}{|l|c|c|}
\hline Qualificatifs caractérisant la communication & Nh. cit. & Fréq. \\
\hline $\begin{array}{l}\text { Complexe, évolutive. diversifiéc. transversale. } \\
\text { géométrie variable }\end{array}$ & 3.5 & $25 \%$ \\
\hline Stratégique, indispensable. réactive & 3.3 & $24 \%$ \\
\hline $\begin{array}{l}\text { Eprouvante. difficile, stressante. } \\
\text { décourageante, marginale, récente }\end{array}$ & 27 & $19 \%$ \\
\hline Relationnelle/humaine, pédagogique. fédératrice & 17 & $12 \%$ \\
\hline $\begin{array}{l}\text { Intéressante, passionnante, enrichissante. } \\
\text { valorisante. créatrice }\end{array}$ & 1.5 & $11 \%$ \\
\hline $\begin{array}{l}\text { Pertinente, rapide. ciblée. instrumentalisée. } \\
\text { concrète/pratique }\end{array}$ & 1.3 & $9 \%$ \\
\hline TOTAL CIT. & 140 & $100 \%$ \\
\hline
\end{tabular}

Les activités de communication sont également présentées comme transverses, ce qui renvoie bien à leur rôle d'une part, d'intervention interne auprès de l'ensemble des personnels et des services et, d'autre part, de transmission à l'externe de messages ayant une valeur identitaire pour l'organisme. Le terme de complexité est assez peu associé à la notion de difficultés. Les entretiens montrent que les $\mathrm{CC}$ définissent de cette manière des activités dont les objets et les méthodologies sont multiples, interactives et imbriquées. Cette idée de transversalité à laquelle nous préférerons celle de complexité, confère cependant un caractère interdisciplinaire à l'activité des $\mathrm{CC}$ et semble accorder un rôle essentiel aux processus de construction du sens au plan interne comme au plan externe de l'institution. En second lieu, les activités de communication sont clairement identifiées 
comme stratégiques, c'est-à-dire ici comme devant assumer des rôles forts d'orientation, de représentation, de clarification, de fédération, ainsi que de mise à disposition de services nécessitant une information et une pédagogie les rendant accessibles et attractifs parce que correspondant à la réalité de vie, souvent difficile, des destinataires des prestations familiales. Il est clair que la communication apparaît ici comme l'un des vecteurs des réponses des $\mathrm{Caf}$ face à la demande sociale.

51 C'est donc globalement une image riche et positive qui prédomine vis-à-vis d'un métier inscrit dans la complexité des organisations et du travail, et ceci quelles que soient les difficultés non négligeables de positionnement et d'identité qui sont mentionnées comme des épreuves à franchir par les CC.

\section{En guise de conclusion provisoire : une professionnalité en émergence}

Les résultats obtenus et analysés au cours de cette recherche renvoient à plusieurs problématiques relatives à la fonction communication au sein des CAF mais aussi à la place et au rôle attribués à celle-ci au sein des administrations françaises.

Tout d'abord, un certain flou, parfois des confusions, règnent en Caf, comme dans bien d'autres institutions sans doute, à propos des métiers de la communication, en particulier au plan du positionnement et de l'identification d'activités dont certaines relèvent d'une fonction de technicien(ne) ou d'assistant(e) et d'autres davantage d'une fonction d'encadrement. Ce flou, certes relatif mais néanmoins avéré, est présent même lorsque la position statutaire des intéressés peut être identifiée. Il questionne le besoin d'une certaine (re)clarification du travail prescrit, des missions, des rôles ainsi que des modes d'évaluation de la fonction communication.

Par là même et à travers l'ensemble des résultats, apparaît un important besoin de professionnalisation des CC. Nous entendons par professionnalisation le passage de pratiques, plus ou moins formalisées et théorisées, à une profession capable de déclarer socialement et de pérenniser son identité, sa technicité, sa culture et ses contributions (Dubar. 1991). Cette conclusion ne doit pas être interprétée comme une absence de savoirs professionnels chez les CC. Elle signifie bien que les acteurs de cette fonction disposent d'un certain nombre de savoirs construits préalablement ou bien à travers leur action quotidienne et les formations qu'ils ont reçues. Cependant, ce sont des savoirs de processus qui leur font souvent défaut, que ce soit pour des activités à dominante technologique ou des activités d'organisation, de relation ou de management. Ainsi, des compétences en matière d'analyse/diagnostic, de problématisation, de conception stratégique de messages ou de projets, de pilotage par l'évaluation, de découverte et d'adaptabilité à différentes cultures d'interlocuteurs, de prises d'information et de capacité à recommander, préconiser, sont évoquées à plusieurs reprises dans les dires des CC. Ces compétences semblent constituer les fondements méthodologiques et culturels permettant de contexlualiser, réassurer et développer les savoirs d'action construits par ailleurs, souvent par l'expérience et la recherche personnelle. La dimension «Chef de projet » est relativement inhérente aux fonctions de communication et concerne tant des aspects techniques qu'organisationnels inscrits dans la recherche d'une intersubjectivité. Elle signifie surtout la construction d'une capacité à approcher de façon systémique et non pas éparpillée ou disparate, un message, un dossier ou un projet en communication, 
activités complexes par définition et ceci, quel que soit le niveau d'intervention. C'est donc la possibilité de reconceptualiser et de reproblémaliser leur métier qui semble jouer un rôle clé dans cette perspective de professionnalisation.

Cette démarche, dont les $\mathrm{CC}$ perçoivent intuitivement l'intérêt en l'exprimant en filigrane dans leurs propos, interroge le sens attribué à la fonction de communication dans les Caf, comme dans d'autres organisations. C'est la lisibilité des métiers liés à la communication qui se trouve questionnée en termes d'identification et de compréhension d'activités, de champs d'intervention et de compétences, mais aussi de résultats attendus et de positionnement institutionnel, donc de valeur sociale de la communication dans les organisations. Cette dernière demeure assez fréquemment vécue comme un risque, notamment par l'encadrement. En effet, du fait qu'elle interroge le sens, la pertinence et la portée de l'action, elle constitue une dimension intermédiaire et interdisciplinaire dans les processus de décision et de pilotage et vient ainsi ébranler les certitudes, les habitudes et les territoires que la notion même d'organisation et son substrat, le management, cherchent à instaurer. Ainsi, les intentions de la communication et de ceux qui en font leur métier, paraissent-elles floues à bon nombre d'interlocuteurs. Cette indistinction de ses finalités se transforme parfois en rejet dès que pointe le sentiment que de surcroît, la communication aurait la possibilité de lire et de mettre à jour les intentions des autres fonctions de l'organisation et de leurs acteurs... La communication semble donc se situer, ou plutôt être située, au sein d'un imaginaire où prédomine le risque au regard de l'habituelle prévisibilité fonctionnelle recherchée par l'administration et le management.

Il faut encore s'interroger sur la promesse que peut porter la fonction communication, promesse vécue très fortement par l'encadrement, les administrateurs et les autres services en cas de coup dur, de difficulté, dans ces moments où l'on attend d'elle que, comme par magie, elle sache inverser une situation délicate et ses effets négatifs sur l'image de l'organisation. On retrouve dans ces instants le mythe d'une communication qui échapperait à toute réalité, comme vouée à osciller entre l'art et le mystère, condamnée à réussir pour, très vite, se rendre discrète et se faire oublier jusqu'au prochain dérapage qu'il lui sera demandé de contrôler. De ce point de vue. la problématique de la promesse affichée ou attribuée à la communication, renvoie à l'interrogation du degré d'information et de formation des personnels d'encadrement et de direction des administrations à ce sujet.

Les activités des $\mathrm{CC}$ vivent des mutations importantes, notamment au plan technologique, mais pas seulement. Une pédagogie de ces changements paraît relativement absente dans l'administration et place les CC dans un certain embarras identitaire et fonctionnel. En particulier, la forte hétérogénéité constatée au plan des savoir-faire et du mode d'utilisation des nouvelles technologies montre des difficultés d'appropriation et de capacité à «travailler autrement ». La gestion du temps, l'efficience dans l'organisation des activités, la prise en charge des demandes et des commandes, les recommandations et préconisations, peuvent se trouver affectées par le sentiment excessif de travailler dans l'urgence sous le joug d'une forte charge mentale et sans visibilité ni validation suffisantes quant aux effets attendus de l'intervention de la communication, y compris sous ses aspects technologiques. De nombreuses personnes interrogées ont également évoqué leur besoin de se construire une "culture de la communication " qu'ils jugent nécessaire pour la compréhension et le traitement des situations auxquelles elles sont confrontées. Sur ce plan, le caractère interdisciplinaire et complexe de la communication, déjà évoqué ici, vient-il se heurter à la prégnance d'une culture administrative où 
prédominent les corps, les grades et les statuts. Il parait pourtant ruineux d'envisager un meilleur positionnement de la fonction communication dans l'administration par le truchement d'un éventuel renoncement des institutions à cet état de fait structurel et symbolique.

L'émergence de la professionnalité de responsable de la communication constitue plutôt, dans les Caf, comme dans d'autres organisations, une problématique d'innovation, ici liée à un métier. En cela, elle implique l'existence d'un terreau favorable à son développement (Aller, 1996) et nous avons vu ici à quel point le rôle de l'encadrement, en tant que relais et porteur du changement, est essentiel sur ce plan. Ensuite, un enjeu est clairement apparu au cours de cette recherche: celui relatif au développement d'une culture de communication mieux considérée et mieux intégrée par ceux qui exercent une responsabilité à ce sujet. Un meilleur cadrage conceptuel et méthodologique, laissant une plus large place à des questions de processus par rapport à des aspects de procédures, paraît constituer la base d'un partage et d'une mutualisation de savoirs et d'expériences propices à la reconnaissance sociale d'un véritable métier. Cette ambition viserait à donner à la notion de réseau de responsables de communication une véritable signification et un caractère de novation au-delà de son seul aspect fonctionnel, souvent limité en réalité à des échanges ponctuels. Enfin, la fonction communication et l'activité de ses responsables semblent inviter les organisations qui les abritent. à «travailler autrement ", notamment en termes d'intercompréhension et de coopération. Il ne s'agit sans doute pas du moindre défi lancé à la fonction communication, celui d'occuper une place reconnue dans les administrations, tout en contribuant à leur transformation sociale au service de l'usager.

\section{BIBLIOGRAPHIE}

ALTER N., Sociologie de l'entreprise et de l'innovation. Paris : L'Harmattan. 1996.

BARBIER J-M. et al., Savoirs théoriques et savoirs d'action. Paris : PUF, 1996.

BONNET J., Le management par projet à l'épreuve des différences sociocognitives de ses acteurs. Communication et Organisation. GREC/O, Université Michel de Montaigne, Bordeaux, 1998 a. pp. 158-173.

BONNET R., L'émergence de la communication dans les métiers exercés par les ingénieurs, L'Orientation Scolaire et Professionnelle. 2001.

BRUNER J., Savoir faire, savoir dire. Paris : PUF. 1983.

CAMILLERI C, Chocs de cultures, concepts et enjeux pratiques de l'interculturel, Paris : L'Harmattan, 1989.

CAMILLERI C. VINSONNEAU G., Psychologie et culture. Paris : Armand Colin, 1996.

CLANET C, L'interculturel - Introduction aux approches interculturelles en Education et en Sciences humaines, Toulouse : Presse Universitaire du Mirail. 1993.

DOISE W., Logiques sociales dans le raisonnement, Neuchâtel/Paris : Delachaux et Niestlé, 1993. 
DUBAR C. La sociologie, Paris, 1991.

DUBAR C, Tripier P, Sociologie des professions, Paris : Armand Colin, 1998.

JODELET D.. Les représentations sociales, Paris : PUF, 1989. WOLTON D., Penser la communication, Paris : Flammarion, 1997.

ZARIFIAN P., Travail et communication, Paris : PUF, 1996

\section{NOTES}

1. Marronniers : activités cycliques des Caf, telles que l'aide aux allocataires au moment de la déclaration des revenus ou encore le traitement des demandes de prêts par les étudiants de juin à septembre.

\section{RÉSUMÉS}

Cette recherche a pour objectif de caractériser une fonction en émergence dans le contexte de l'administration française, ici celle de Chargé de communication au sein des Caisses d'Allocations Familiales. Les investigations réalisées ont permis de mettre en évidence les activités, les contraintes, les questions identitaires qui représentent la problématique de constitution et de développement d'un métier à part entière que les organisations reconnaissent parfois encore difficilement. Les connaissances produites ici visent à contribuer à la constitution d'un cadre théorique et d'une démarche méthodologique en vue de l'analyse des métiers liés à la communication dans des organisations de plus en plus interpellées par les exigences de la demande sociale.

The purpose of this research is to define a rising position in the context of the French administration, namely that of communications manager within the Family Allowance Office. Investigations carried out highlight the activities, the pressures and the identity issues representative of the problems linked to the birth and the development of this position. The latter is a real job but a position yet hardly recognised by the organisations. The knowledge brought up here, aims at contributing to the building of a theoretical framework and a method. Both of them will help us analysing communication positions in the organisations that are more and more questioned by the requirements of the social demand.

\section{INDEX}

Mots-clés : chargés de communication, Dircom, Professionnalité, compétences 


\section{AUTEURS}

\section{ROSETTE BONNET}

Rosette et Jacques Bonnet sont respectivement Maître de conférences et Professeur au Département des Sciences de la Formation et de la Communication de l'ENESAD

(Etablissement National d'Enseignement Supérieur Agronomique de Dijon). Leurs travaux portent sur la communication organisationnelle, notamment en termes d'analyse des modèles de changement au sein des administrations et des entreprises. Ils s'intéressent également à la constitution et au développement de nouvelles professionnalités dans le champ de la communication.

\section{JACQUES BONNET}

Jacques Bonnet est directeur de recherches à l'Université de Bourgogne (LIMSIC). 